\title{
A Novel Digital Watermarking Algorithm using Random Matrix Image
}

\author{
Mahimn Pandya \\ Smt. K.B.Parekh College of \\ Computer Science, \\ Bhavnagar University.
}

\author{
Hiren Joshi \\ Department of Computer \\ Science, Gujarat University.
}

\author{
Ashish Jani \\ Department of Computer \\ Science, Kadi Sarva \\ Vishwavidhyalay
}

\begin{abstract}
The availability of bandwidth for internet access is sufficient enough to communicate digital assets. These digital assets are subjected to various types of threats. [19] As a result of this, protection mechanism required for the protection of digital assets is of priority in research. The threat of current focus is unauthorized copying of digital assets which give boost to piracy. This under the copyright act is illegal and a robust mechanism is required to curb this kind of unauthorized copy. To safeguard the copyright digital assets, a robust digital watermarking technique is needed. The existing digital watermarking techniques protect digital assets by embedding a digital watermark into a host digital image. This embedding does induce slight distortion in the host image but the distortion is usually too small to be noticed. At the same time the embedded watermark must be robust enough to with stand deliberate attacks. There are various techniques of digital watermarking but researchers are making constant efforts to increase the robustness of the watermark image. The layered approach of watermarking based on Huffman coding [5] can soon increase the robustness of digital watermark.[11] Ultimately, increasing the security of copyright of protection. The proposed work is in similar direction where in RMI (Random Matrix Image) is used in place of Huffman coding. This innovative algorithm has considerably increased the robustness in digital watermark while also enhancing security of production.
\end{abstract}

Keywords: Digital Watermarking, Random Matrix Image, Image Processing, Embedding, Extraction

\section{INTRODUCTION}

The Internet usage to transfer electronic assets has involved a technique that is able to protect the copyright of published medias into a certainty. The easy distribution of these documents through the web may violate protection laws against unauthorized copies and make fidelity questionable. Digital watermarking has been projected as a solution against these practices. Digital watermark is an authenticating technique of digital data with secret information that can be extracted to the receptor. The image in which this data is inserted is called 'cover image' or 'host image'. The watermarking process has to be resistant against possible attacks, keeping the content of the watermark readable in order to be recognized when extracted. Features like robustness and fidelity are essentials of a watermarking system however the size of the embedded information has to be considered since data becomes less robust as its size increases. Therefore a trade-off of these features must be considered. [1, 6, 10, 16 and 17]

\section{PRINCIPLE OF DIGITAL WATERMARKING}

A digital watermarking process have three phases, first embedding, second attack and third detection. In Embedding, an algorithm accepts the host image and the watermark image or data to be embedded and produces a watermarked image. The watermarked image is transmitted or stored, usually transmitted to another person. If this person makes a modification, this is called an attack. [12, 13, $14,15,20,21]$ There are various kinds of attacks like copy, removal, mosaic etc. Watermark detection is an algorithm which is used to find the attacked data to attempt to extract the watermark from it. If the watermarked image is not modified during transmission, then the watermark is still present and it can be extracted. If the watermarked image is copied, then the information is also carried in the copy. The embedding takes place by manipulating the content of the digital data, which means the information is not embedded in the frame around the data, but it is carried with the watermarked image itself. [2] Figure 1 and Figure 2 shows the flowchart of watermarking process.

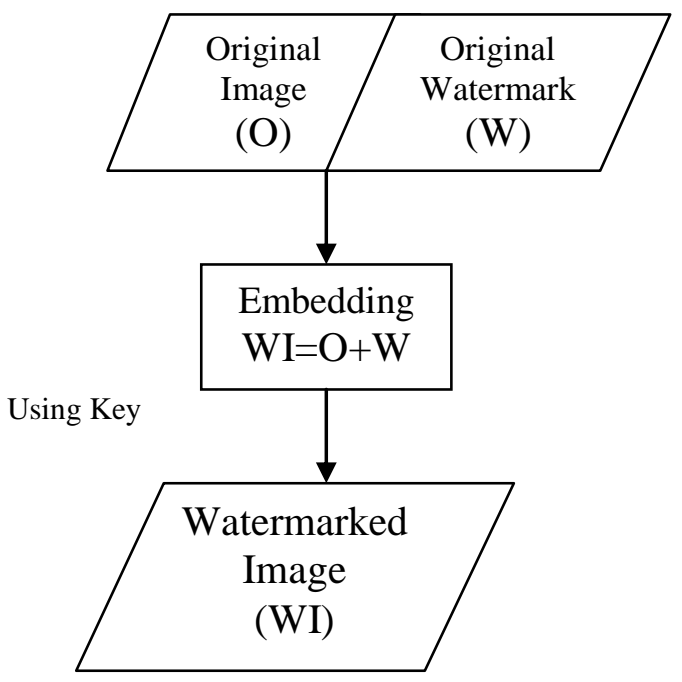

Fig.1 Flowchart of Embedding of Watermark 


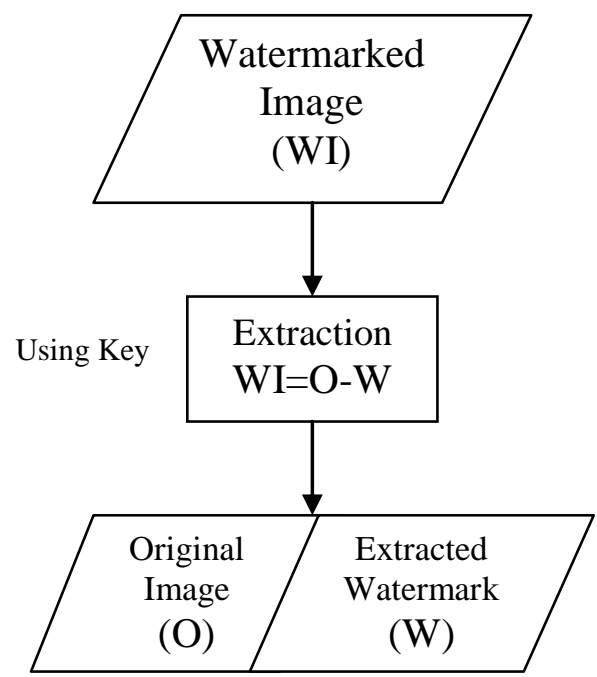

Fig.2 Flowchart of Extraction of Watermark

The original image and the watermark are embedded using one of the watermarking schemes that are available.[8,9] The watermarked image is processed through a detector in which generally a reverse process to that used during the embedding phase is applied to retrieve the watermark. The different watermarking algorithms differ in the way in which it embeds the watermark on to the cover object. A secret key is used during the embedding and the extraction process in order to prevent illegal access to the watermark [2, 22, and 7]. This paper deals with the new watermarking technique which helps to protect digital image based on RMI (Random Matrix Image).

\section{PROPOSED WORK}

In this work we are embedding Random Matrix Image as a watermark. We generate a new watermark image for each and every new image for watermarking. The proposed algorithms are used for watermark embedment and extraction. in watermark embedding we used a RMI to generate unique watermark and this watermark is being used to embed to an digital image. The exact reverse process is used to extract watermark for an image. For extraction we require RMI or an original image.

\subsection{Overview of random matrix image.}

This is an auto generated Image based on Random Matrix Image generated in SCILAB using random function [3]. In SCILAB Random Matrix can be generated using random function having randomised number from given range. In simulation we can also generate real number. For example we want to generate a random matrix of $8 \times 8$ from 0 to 10 numbers. Matrix may cover any number from 0 to 10 like shown in figure 3 (b).

\subsection{Watermark embedding algorithm}

Step1: Read the original image.

Step2: Generate RMI (in range of 0 to 10 ) which is to be embedded. (Secret Key Matrix)

Step3: Add this Generated Image and Original Image in matrix addition form
Step4: Now generate image from matrix form.

Step5: The output image is a watermarked image.

\subsection{Watermark extraction algorithm}

Step1: Read the watermarked image.

Step2: Read matrix (a secret key) which is sent with image.

Step3: Subtract Matrix from watermarked Image in matrix subtraction form.

Step4: Now generate two different images from theses matrices form.

Step5: The output images are Original image and watermarked image.

\section{IMPLIMENTATION \& RESULTS}

SCILAB simulations are performed by using $256 \times 256$ pixel gray level "Lena" image and $256 \times 256$ pixel watermark (RMI)[4]. Figure 3. (a) and (b) Shows greyscale image of 256 X256 pixel and RMI Watermark respectively. Figure 4 (a) and (b) show 8X8 pixel matrix of Figure 3 (a) and (b) images respectively. Figure 5 (a) and (b) shows output image and $8 \times 8$ matrix of .watermarked Lena image.

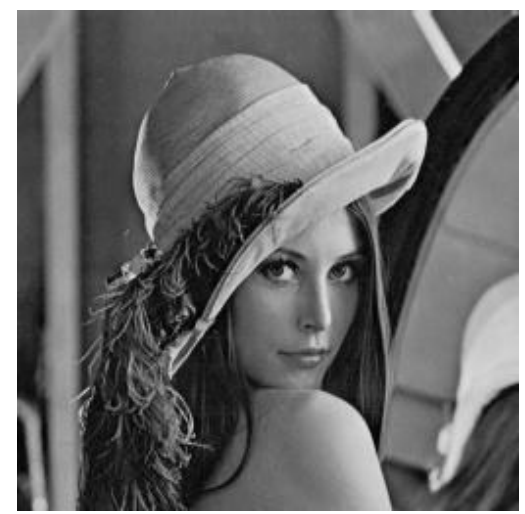

(a)

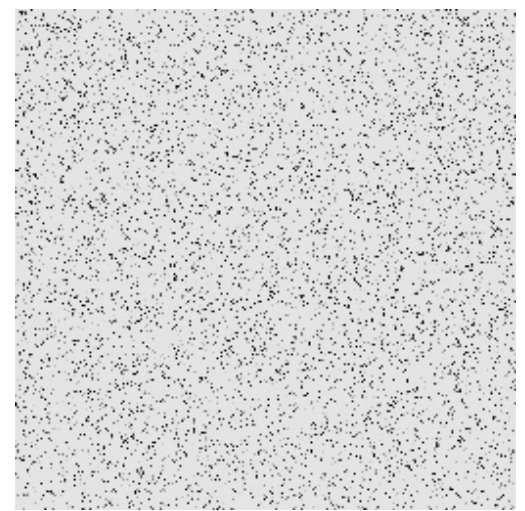

(b)

Fig. 3 (a) Original Lena Image (b) RMI watermark 


\begin{tabular}{llllllll}
\hline 195 & 195 & 196 & 197 & 197 & 198 & 199 & 199 \\
196 & 196 & 196 & 197 & 197 & 197 & 198 & 198 \\
197 & 197 & 197 & 197 & 196 & 196 & 196 & 196 \\
199 & 198 & 198 & 197 & 196 & 195 & 194 & 194 \\
199 & 198 & 197 & 196 & 195 & 194 & 193 & 193 \\
198 & 198 & 197 & 196 & 195 & 194 & 193 & 193 \\
197 & 196 & 196 & 195 & 195 & 194 & 194 & 194 \\
196 & 196 & 195 & 195 & 195 & 194 & 194 & 194 \\
\hline
\end{tabular}

(a)

\begin{tabular}{|llllllll|}
\hline 2 & 2 & 9 & 8 & 2 & 6 & 3 & 6 \\
\hline 7 & 2 & 0 & 0 & 0 & 0 & 8 & 3 \\
\hline 9 & 1 & 3 & 0 & 4 & 0 & 1 & 1 \\
\hline 2 & 6 & 3 & 0 & 7 & 2 & 4 & 2 \\
\hline 9 & 3 & 0 & 6 & 3 & 6 & 7 & 2 \\
\hline 5 & 5 & 3 & 7 & 7 & 0 & 5 & 2 \\
\hline 5 & 1 & 0 & 7 & 5 & 8 & 0 & 5 \\
\hline 2 & 0 & 5 & 5 & 9 & 7 & 6 & 6 \\
\hline
\end{tabular}

(b)

Fig.4 (a) 8x8 matrix of Lena (b) $8 \times 8$ matrix of RMI

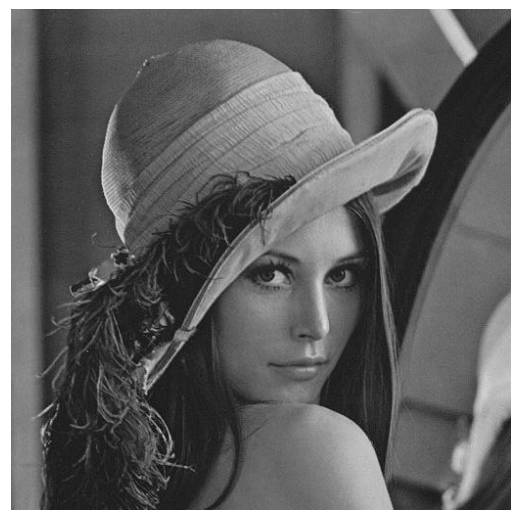

(a)

\begin{tabular}{|llllllll|}
\hline 197 & 197 & 205 & 205 & 199 & 204 & 202 & 205 \\
\hline 203 & 198 & 196 & 197 & 197 & 197 & 206 & 201 \\
\hline 206 & 198 & 200 & 197 & 200 & 196 & 197 & 197 \\
\hline 201 & 204 & 201 & 197 & 203 & 197 & 198 & 196 \\
\hline 208 & 201 & 197 & 202 & 198 & 200 & 200 & 195 \\
\hline 203 & 203 & 200 & 203 & 202 & 194 & 198 & 195 \\
\hline 202 & 197 & 196 & 202 & 200 & 202 & 194 & 199 \\
\hline 198 & 196 & 200 & 200 & 204 & 201 & 200 & 200 \\
\hline
\end{tabular}

(b)

Fig.5 (a) Watermarked Image (b) 8x8 matrix of Fig.5(a)

In figure 5 (a) shows that watermarked Lena image and (b) shows $8 \times 8$ matrix of watermarked the image. Figure. 5(b) surely shows the addition of two matrices shown in image Figure. 4 (a) and (b). The change in pixel vales that shows embedment of RMI to Lena image.

\section{CONCLUSION}

A novel method of digital watermarking based on embedding matrix as a watermark is presented in this paper. The experiment part has used random matrix as a watermark to prevent an attacker for easy attack on the water mark image. The reason for this is that each image usually has different matrix form with range from 0 to 10 . The noticeable part of this work is the use of RMI transition to authenticated user of the image. Without having RMI, none other than authenticated user can detect and extract watermark from watermarked image. The limitation of the current work is that the stated technique of digital water marking is used on grey scale image and not on the coloured image. The experiment performed on greyscale image having less than 245 greyscale pixel value. The extension of this work can cover the use of stated technique of digital water marking on colour images.

\section{REFERENCES}

[1] W. C. H. Fung, G. Antonio, and W. Godoy, "A Review Study on Image Digital Watermarking," The Tenth International Conference on Networks-ICN, pp. 24 28, 2011.

[2] X. Wu and Zhi-Hong Guan, "A novel digital watermark algorithm based on chaotic maps," Physics Letters A, Elsevier, vol. 365, no. 5-6, pp. 403-406, Jun. 2007.

[3] Raman, S. (2010). Image Processing Using Scilab, 129.

[4] Galda, H. (2011). Image Processing with Scilab and Image Processing Design Toolbox.

[5] D. L. Bhaskari, P. S. Avadhani, and M. Viswanath, "A Layered Approach for Watermarking In Images Based On Huffman Coding," International Journal on Computer Science and Engineering, vol. 02, no. 02, pp. 149-154, 2010.

[6] I. J. Cox, M. L. Miller, J. A. Bloom, J. Fridrich, and T. Kalker, Digital Watermarking and Steganography, Second Edi. Morgan Kaufmann Publishers, Elsevier, 2008.

[7] R. L. de Queiroz, "Processing JPEG-compressed images and documents.," IEEE transactions on image processing: a publication of the IEEE Signal Processing Society, vol. 7, no. 12, pp. 1661-72, Jan. 1998.

[8] M. Barni, F. Bartolini, V. Cappellini, and A. Piva, "A DCT-domain system for robust image watermarking," Signal Processing, vol. 66, no. 3, pp. 357-372, May 1998.

[9] D. Kundur and D. Hatzinakos, "Improved robust watermarking through attack characterization.," Optics express, vol. 3, no. 12, pp. 485-90, Dec. 1998. 
[10] X. Qi and J. Qi, “A robust content-based digital image watermarking scheme," Signal Processing, Elsevier, vol. 87, no. 6, pp. 1264-1280, Jun. 2007.

[11] H. Kostopoulos, S. Kandiliotis, I. Kostopoulos, and M. Xenos, "A Digital Image Watermarking Technique Using Modulated Pascal' S Triangles," International Conference Signal Processing, Pattern Recognition \& Applications, pp. 82-86, 2003.

[12] M. A. Suhail, M. S. Obaidat, S. S. Ipson, and B. Sadoun, "A comparative study of digital watermarking in JPEG and JPEG 2000 environments," Information Sciences, Elsevier, vol. 151, pp. 93-105, May 2003.

[13] M. Sreerama Murty, D. Veeraiah, and a Srinivas Rao, "Digital Signature and Watermark Methods For Image Authentication using Cryptography Analysis," Signal \& Image Processing : An International Journal, vol. 2, no. 2, pp. 170-179, Jun. 2011.

[14] D. Kirovski and F. a. P. Petitcolas, "Blind pattern matching attack on watermarking systems," IEEE Transactions on Signal Processing, vol. 51, no. 4, pp. 1045-1053, Apr. 2003.

[15] V. M. Potdar, S. Han, and E. Chang, "A survey of digital image watermarking techniques," INDIN '05. 2005 3rd IEEE International Conference on Industrial Informatics, 2005., no. Indin, pp. 709-716, 2005.

[16] J.-L. Liu, D.-C. Lou, M.-C. Chang, and H.-K. Tso, "A robust watermarking scheme using self-reference image," Computer Standards \& Interfaces, Elsevier, vol. 28, no. 3, pp. 356-367, Jan. 2006.
[17] P. L. Lin, C.-K. Hsieh, and P.-W. Huang, "A hierarchical digital watermarking method for image tamper detection and recovery," Pattern Recognition, vol. 38, no. 12, pp. 2519-2529, Dec. 2005.

[18] G. Bhatnagar and B. Raman, "A new robust reference watermarking scheme based on DWT-SVD," Computer Standards \& Interfaces, Elsevier, vol. 31, no. 5, pp. 1002-1013, Sep. 2009.

[19] S. Radharani and M. L. Valarmathi, "A Study on Watermarking Schemes for Image Authentication," International Journal of Computer Applications, vol. 2, no. 4, pp. 24-32, May 2010.

[20] T. Furon and P. Duhamel, "An asymmetric watermarking method," IEEE Transactions on Signal Processing, vol. 51, no. 4, pp. 981-995, Apr. 2003.

[21] M. Wu and B. Liu, "Attacks on digital watermarks," Conference Record of the Thirty-Third Asilomar Conference on Signals, Systems, and Computers (Cat No.CH37020), vol. 2, pp. 1508-1512.

[22] C.-C. Lai, H.-C. Huang, and Cheng-Chih Tsai, "A Digital Watermarking Scheme Based On Singular Value Decomposition And Micro-Genetic Algorithm" International Journal of Innovative Computing, Information and Control, vol. 5, no. 7, p. 1867-1873, 2009. 